\title{
Promoter methylation and large intragenic rearrangements of DPYD are not implicated in severe toxicity to 5 -fluorouracil-based chemotherapy in gastrointestinal cancer patients
}

\author{
Joana Savva-Bordalo ${ }^{1,4}$, João Ramalho-Carvalho 2,4 , Manuela Pinheiro ${ }^{2,5}$, Vera L Costa ${ }^{2,4}$, Ângelo Rodrigues ${ }^{3}$, \\ Paula C Dias ${ }^{3}$, Isabel Veiga ${ }^{2,5}$, Manuela Machado ${ }^{1}$, Manuel R Teixeira ${ }^{2,5,6}$, Rui Henrique ${ }^{3,4,6}$, Carmen Jerónimo , $^{2,4,6^{*}}$
}

\begin{abstract}
Background: Severe toxicity to 5-fluorouracil (5-FU) based chemotherapy in gastrointestinal cancer has been associated with constitutional genetic alterations of the dihydropyrimidine dehydrogenase gene (DPYD).

Methods: In this study, we evaluated DPYD promoter methylation through quantitative methylation-specific PCR and screened DPYD for large intragenic rearrangements in peripheral blood from 45 patients with gastrointestinal cancers who developed severe 5-FU toxicity. DPYD promoter methylation was also assessed in tumor tissue from 29 patients

Results: Two cases with the IVS14+1G > A exon 14 skipping mutation (c.1905+1G > A), and one case carrying the $1845 \mathrm{G}>$ T missense mutation (c.1845G > T) in the DPYD gene were identified. However, DPYD promoter methylation and large DPYD intragenic rearrangements were absent in all cases analyzed.

Conclusions: Our results indicate that DPYD promoter methylation and large intragenic rearrangements do not contribute significantly to the development of 5-FU severe toxicity in gastrointestinal cancer patients, supporting the need for additional studies on the mechanisms underlying genetic susceptibility to severe 5-FU toxicity.
\end{abstract}

\section{Background}

The fluoropyrimidine 5-fluorouracil (5-FU) is broadly used in the treatment of a wide range of gastrointestinal cancers[1]. 5-FU is an analogue of uracil with a fluorine atom at the $\mathrm{C}-5$ position replacing hydrogen, which enters the cell using the same transport machinery as the uracil nucleotide[2]. The mechanism of 5-FU cytotoxicity has been ascribed to the inhibition of thymidylate synthase (TYMS) and misincorporation of fluoronucleotides into RNA and DNA $[1,3]$. The ratelimiting enzyme in 5-FU catabolism is dihydropyrimidine dehydrogenase (DPD), which converts 5-FU to dihydrofluorouracil. More than $80 \%$ of administered 5 -FU is normally catabolized primarily in the liver, where DPD is abundantly expressed[1].

\footnotetext{
* Correspondence: carmenjeronimo@ipoporto.min-saude.pt

${ }^{2}$ Department of Genetics, Portuguese Oncology Institute - Porto, Portugal Full list of author information is available at the end of the article
}

The types of toxicity associated with 5-FU are predominantly myelosuppression, diarrhea, mucositis, and hand-foot syndrome[4,5]. The mechanism of 5-FU cytotoxicity may depend on genetic and clinical factors. Female gender and mode of administration in bolus are linked to increased toxicity[6]. In 5-FU bolus schedules, the incorporation of fluorouridine triphosphate into RNA appears to be the most important mechanism of action, whereas when the infusion time is prolonged, inhibition of TYMS becomes more important resulting in lower toxicity[7].

The expression levels of DPD and TYMS vary among individuals and may be related to different toxicity profiles. These might also be potentially important as prognostic factors and predictive markers of response to 5 -FU[8,9]. However, no reliable molecular marker of sensitivity, resistance, or toxicity to 5 -FU has been validated until now[10]. However, partial or complete DPD 
deficiency is a well known pharmacogenetic syndrome, detected in $3 \%$ to $5 \%$ of the general population, which has been associated with severe and potentially lethal toxicity following 5-FU administration[11].

The dihydropyrimidine dehydrogenase gene (DPYD), which codes for DPD, is present as a single copy gene on chromosome $1 \mathrm{p} 22$ and consists of 23 exons. Although more than 50 gene alterations have been characterized during the past decade[12], the majority of them represents missense or intronic variants with unknown biological and clinical significance $[12,13]$. Indeed, only a limited number of patients are carriers of allelic variants (including the most prevalent exon 14 skipping mutation, IVS14+1G > A) that significantly affect DPD catalytic activity[14]. Recently, large intragenic rearrangements of $D P Y D$ and a new interstitial deletion [del(1)(p13.3p21.3)] were found in some DPD deficient patients[15].

Nevertheless, the genetic variants reported thus far do not account for most of the DPD deficiency cases. Thus, epigenetic de-regulation of DPYD was hypothesized as an alternative mechanism for reduced DPD activity. In this setting, Noguchi and co-workers found that DPD activity was controlled at the transcriptional level by promoter methylation and thus aberrant methylation might affect the sensitivity to 5-FU in hepatocarcinoma cell lines[16]. Subsequently, Ezzeldin et al. assessed DPD activity and DPYD promoter methylation status in a small series of clinical samples $(n=15)$ from normal individuals and cancer patients [17]. DPYD promoter methylation was detected in peripheral bloods samples from all (five) DPD-deficient volunteers and in three out of five DPD-deficient cancer patients with a previous history of 5-FU toxicity. Interestingly, no evidence of methylation was detected in samples from five volunteers with normal DPD activity[17]. Finally, methylation of DPYD promoter region of RKO colorectal cancer cell line was shown to be associated with decreased gene expression[18].

We sought to characterize the DPYD promoter methylation status and the presence of large intragenic rearrangements in a series of gastro-intestinal (GI) cancer patients to determine whether these might constitute alternative mechanisms for DPD deficiency and a cause of severe 5-FU toxicity.

\section{Methods}

\section{Study design}

The primary objective of this cross-sectional study was to analyze the methylation status of DPYD promoter region in GI patients who developed severe 5-FU toxicity. The secondary objective was the analysis of large intragenic rearrangements of $D P Y D$.

\section{Patient selection and Clinical evaluation}

Forty-five patients with esophageal, gastric or colorectal cancer (Table 1) who had developed severe toxicity following chemotherapy regimens based on 5-FU and had been tested for DPYD exon 14 mutations (including the exon 14 skipping mutation IVS14+1G > A) by direct sequencing analysis at the Portuguese Oncology Institute - Porto, Portugal, from January 1994 through December 2008. All the patients were enrolled in this study, following informed consent. These studies were approved by the respective institutional review board (Comissão de Ética do IPO-Porto).

Data on patient demographics (gender and age), ECOG, tumor anatomical site, TNM staging, 5-FUbased chemotherapy scheme, mode of administration and toxicity profile were assessed by detailed hospital chart review for each case (Table 1). This study included 45 patients ( 24 women and 21 men). The median age was 56 years (ranging from 34 to 76 years) without differences across gender $(p=0.432)$. The combination of 5 -FU with cisplatinum was given in 23 cases $(51.1 \%)$, which were all esophageal or gastric cancer patients. In the 22 colorectal patients, 5-FU was given in conjunction with levamisole in 2 cases, combined with folinic acid in 12 cases. FolFOx was administered in 6 cases and FolFIri in 2 cases.

\section{Table 1 Clinical and pathological characteristics of} patient population

\begin{tabular}{|c|c|c|}
\hline & & n (\%) \\
\hline No. of patients & & $45(100)$ \\
\hline \multirow[t]{2}{*}{$\begin{array}{l}\text { Gender; Median age } \\
\text { (Min - Max), yrs }\end{array}$} & Female & $\begin{array}{l}24(53.3) ; 54 \\
\quad(34-76)\end{array}$ \\
\hline & Male & $\begin{array}{l}21(46.7) ; 61 \\
\quad(35-75)\end{array}$ \\
\hline \multirow[t]{2}{*}{ ECOG } & 0 & $20(44.4)$ \\
\hline & $\geq 1$ & $25(55.6)$ \\
\hline \multirow[t]{3}{*}{ Tumor Location } & Esophageal & $4(8.9)$ \\
\hline & Gastric & $19(42.2)$ \\
\hline & Colorectal & $22(48.9)$ \\
\hline \multirow[t]{3}{*}{ TNM Stage } & $\|$ & $3(6.7)$ \\
\hline & III & $12(26.7)$ \\
\hline & IV & $30(66.7)$ \\
\hline \multirow[t]{2}{*}{ Treatment Purpose } & Adjuvant & $14(31.1)$ \\
\hline & Palliative & $31(68.9)$ \\
\hline \multirow[t]{2}{*}{ Chemotherapy Scheme } & 5-FU/Cisplatinum & $23(51.1)$ \\
\hline & Other* & $22(48.9)$ \\
\hline \multirow{3}{*}{$\begin{array}{l}5 F U \\
\text { Mode of Administration }\end{array}$} & Bolus alone & $15(33.3)$ \\
\hline & Bolus $+\mathrm{Cl}^{\#}$ & $8(17.8)$ \\
\hline & Continuous infusion & $22(48.9)$ \\
\hline
\end{tabular}

*5-FU/LV or 5-FU/FA or FolFOx or FolFIri; ${ }^{*}$ Continous Infusion 


\section{Toxicity Grade following chemotherapy with 5-FU}

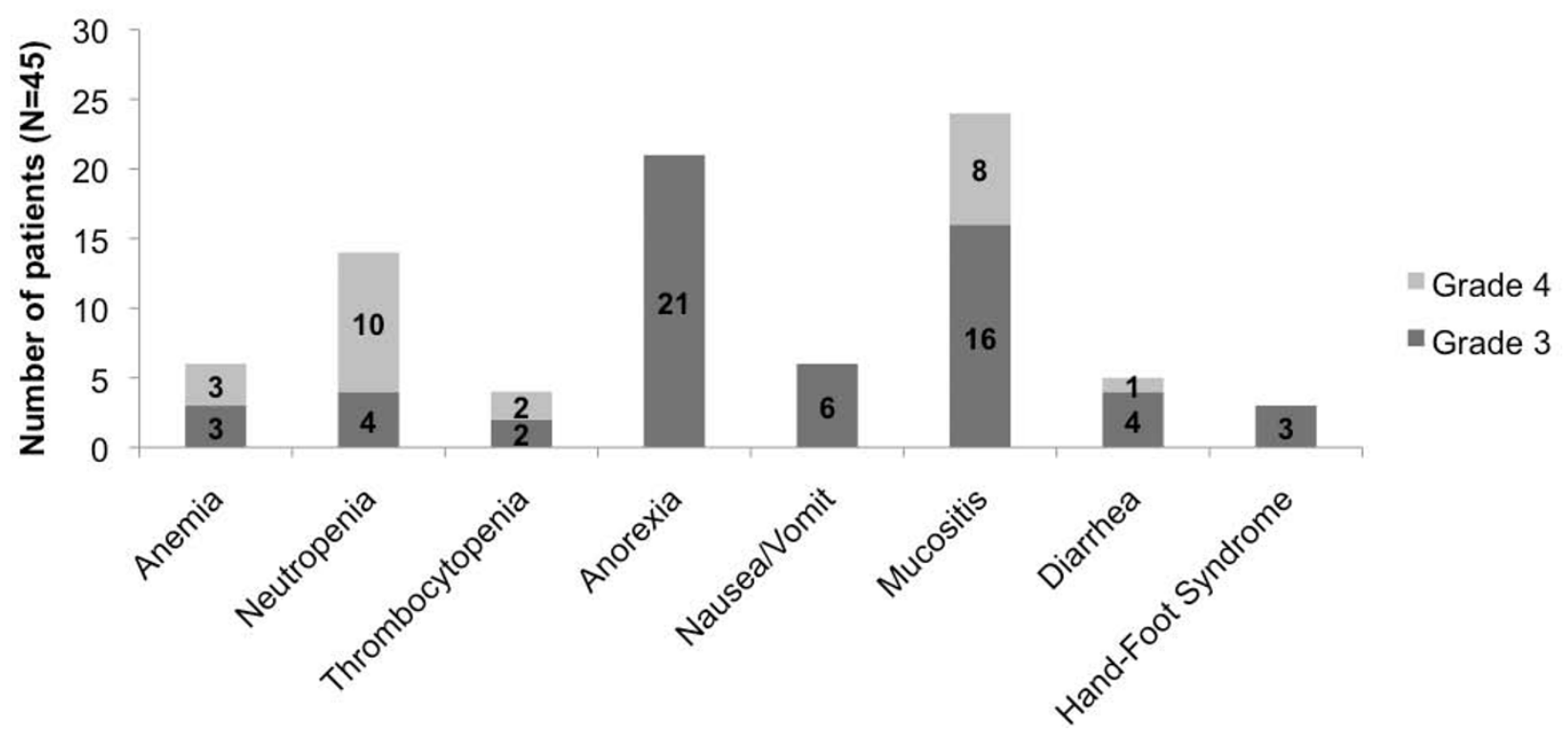

Figure 1 Toxicity profile of the 45 patients with severe toxicity treated with 5-FU according to Common Terminology Criteria for Adverse Events, v4.0.

Adverse drug effect during chemotherapy was classified according to the Common Terminology Criteria for Adverse Events (CTCAE) v4.0[19]. Accordingly, toxicity grades 3 or 4 were considered severe. Patients developed the following manifestations of toxicity grade 3 or 4 during chemotherapy with 5-FU (Figure 1): mucositis (24 cases, 53\%), anorexia ( 21 cases, $47 \%$ ), neutropenia (14 cases, $31 \%)$, anemia (6 cases, 13\%), nausea/vomiting (6 cases, 13\%), diarrhea ( 5 cases, 11\%), thrombocytopenia (4 cases, 9\%) and hand-foot syndrome (3 cases, $7 \%$ ).

The clinicopathological characteristics and toxicity profile of three carriers of DPYD mutations identified in this study are provided in Table 2 .

\section{Sample collection and tissue processing}

A peripheral blood sample was obtained for all patients, and in 29 of these, a tumor-representative formalinfixed, paraffin-embedded tissue block (biopsy or surgical specimen before chemotherapy) was available. Routine histopathological evaluation, comprising diagnosis, grading and pathological staging according to $\mathrm{WHO}[20]$ was performed in all cases. Serial sections were obtained from each of the representative tissue block and an expert pathologist delineated their highest proportion of malignant cells. Colorectal carcinoma cell line RKO from American Type Tissue Collection (ATTC, USA) was used as a positive control for methylation analysis (this cell line is methylated at the DPYD promoter). RKO cells were grown in ATCC-formulated Dulbecco's Modified Eagle Medium.

\section{DNA extraction, quantification and modification}

DNA was extracted from all available samples (RKO cell line, peripheral blood and microdissected tumor tissue) with phenol-chloroform method[21] and quantified using Nanodrop ${ }^{\mathrm{Tu}}$ ND1000 microspectrophotometer (NanoDrop, USA). Genomic DNA extracted from peripheral blood, cell line and microdissected tumor was submitted to sodium bisulfite conversion using a previously described method [22-24]. Briefly, $1 \mu \mathrm{g}$ of genomic DNA from each sample were used for the chemical treatment. Bisulfite-modified DNA was purified using a vacuum manifold and a Wizard DNA Clean-up System [Promega Corp., WI, USA ], treated again with sodium hydroxide, precipitated with ethanol, eluted in $60 \mu \mathrm{l}$ of water and stored at $-80^{\circ} \mathrm{C}$.

\section{Real-time, quantitative, methylation-specific PCR}

The chemically modified DNA from the RKO cell line, peripheral blood and microdissected tumor tissue was amplified through quantitative methylation-specific PCR (QMSP) [25]. Primers were designed according to 
Table 2 Clinicopathological characteristics and toxicity profile of DPYD mutation carriers

\begin{tabular}{|c|c|c|c|}
\hline & Patient \#1 & Patient \#2 & Patient \#3 \\
\hline Age & 35 & 64 & 68 \\
\hline Gender & female & male & female \\
\hline \multicolumn{4}{|l|}{ Tumor } \\
\hline Location & Colorectal & Colorectal & Esophageal \\
\hline Type & Adenocarcinoma & Adenocarcinoma & Squamous cell carcinoma \\
\hline Stage & IV & $\| I \mathrm{~B}$ & IV \\
\hline \multicolumn{4}{|l|}{ Treatment regimen } \\
\hline \multicolumn{4}{|l|}{ Toxicity profile } \\
\hline Mucositis & G3 & G4 & G4 \\
\hline Anemia & G4 & G0 & G0 \\
\hline Neutropenia & G4 & G4 & G0 \\
\hline Thrombocytopenia & G4 & G0 & G0 \\
\hline DPYD mutation type & $1845 \mathrm{G}>\mathrm{T}$ & IVS14+1G > A & IVS14+1G > A \\
\hline
\end{tabular}

the $\mathrm{CpG}$ island at the promoter and exon 1 of the sense strand of the DPYD gene, starting at $-266 \mathrm{bp}$ from transcription start site (Genbank accession no. NM_000110) as follows: forward, 5'-TTTGTTTGT TTTCGATTCGC-3'; and reverse 5'-ATCCGCCGAATCCTTACTAA-3' (amplicon size of 208 bp). A reference gene $(A C T B)$ was used to normalize for DNA input in each sample[26]. Fluorescence based QMSP assays were carried out using the SYBR ${ }^{\oplus}$ Green PCR Master Mix (Applied Biosystems, USA). Running conditions were: $50^{\circ} \mathrm{C}$ for $2 \mathrm{~min}, 95^{\circ} \mathrm{C}$ for $10 \mathrm{~min}$ followed by 45 cycles of $95^{\circ} \mathrm{C}$ for $15 \mathrm{sec}$, and $60^{\circ} \mathrm{C}$ for $1 \mathrm{~min}$. After 45 cycles, a dissociation-curve analysis was performed using the following conditions: $95^{\circ} \mathrm{C}$ for $15 \mathrm{sec}, 60^{\circ} \mathrm{C}$ for $20 \mathrm{sec}$ and $95^{\circ} \mathrm{C}$ for $30 \mathrm{sec}$. Each sample was run in triplicate and, additionally, multiple water blanks were used per plate as a control for contamination (negative control). All amplifications were carried out in 96-well plates on a 7500 Sequence Detection System (Applied Biosystems, USA). In each plate, five serial $10 x$-dilutions of fully methylated, bisulfite converted DNA - CpGenome Universal Methylated DNA [Millipore, CA, USA] - were also included to construct the standard curve in order to quantify the amount of fully methylated alleles in each reaction. A run was considered valid when the following six criteria were met: (1) slopes of each standard curve above -3.60 corresponding to a PCR efficiency $>90 \%$; (2) $\mathrm{R}^{2}$ of at least four relevant data points $\geq 0.99$; (3) no template controls not amplified; (4) the positive methylation control had to provide a methylated signal; (5) the negative control had no signal; and (6) threshold cycle value for each gene $\leq 40$. To determine the relative levels of methylated promoter DNA in each sample, the values obtained by QMSP analysis (mean quantity) for the target gene were divided by the respective values of the internal reference gene
$(A C T B)$. The ratio thus generated, which constitutes an index of the percentage of input copies of DNA that are fully methylated at the primer site, was then multiplied by 1000 for easier tabulation (methylation level $=$ target gene $/$ reference gene $\times 1000)$.

\section{DPYD large genomic rearrangements analysis}

Peripheral blood DNA samples were also screened for $D P Y D$ large genomic rearrangements by Multiplex Ligation Probe Amplification (MLPA) according to the manufacturer's instructions (SALSA P103 kit; MRC, Holland). This probemix contains probes with target-specific sequences for each of the 23 exons and promoter region. Owing to the large size of the introns, probes for five of the introns are also included. The MLPA method is based on sequence-specific probe hybridization to genomic DNA, followed by PCR amplification of the hybridized probe (with one FAM-labeled primer) and semi-quantitative analysis of the PCR products. A deletion or duplication was scored if the relative peak area of the amplification product presented a reduced or augmented area of 35 to $50 \%$ when compared to a normal control, respectively. The kit also contains a probe specific for the exon 14 skipping mutation $($ IVS14+1G $>$ A) that only generates a signal when the mutation is present [27].

\section{Statistical analysis}

Descriptive statistics were used to summarize the clinicopathological, molecular and immunoexpression data. The independent samples Mann-Whitney U test was applied to determine if the age distribution was the same across genders. The analysis was performed with PASW Statistics 18.0 software.

\section{Results}

No evidence of DPYD promoter methylation was observed in any of the 45 peripheral blood samples nor 


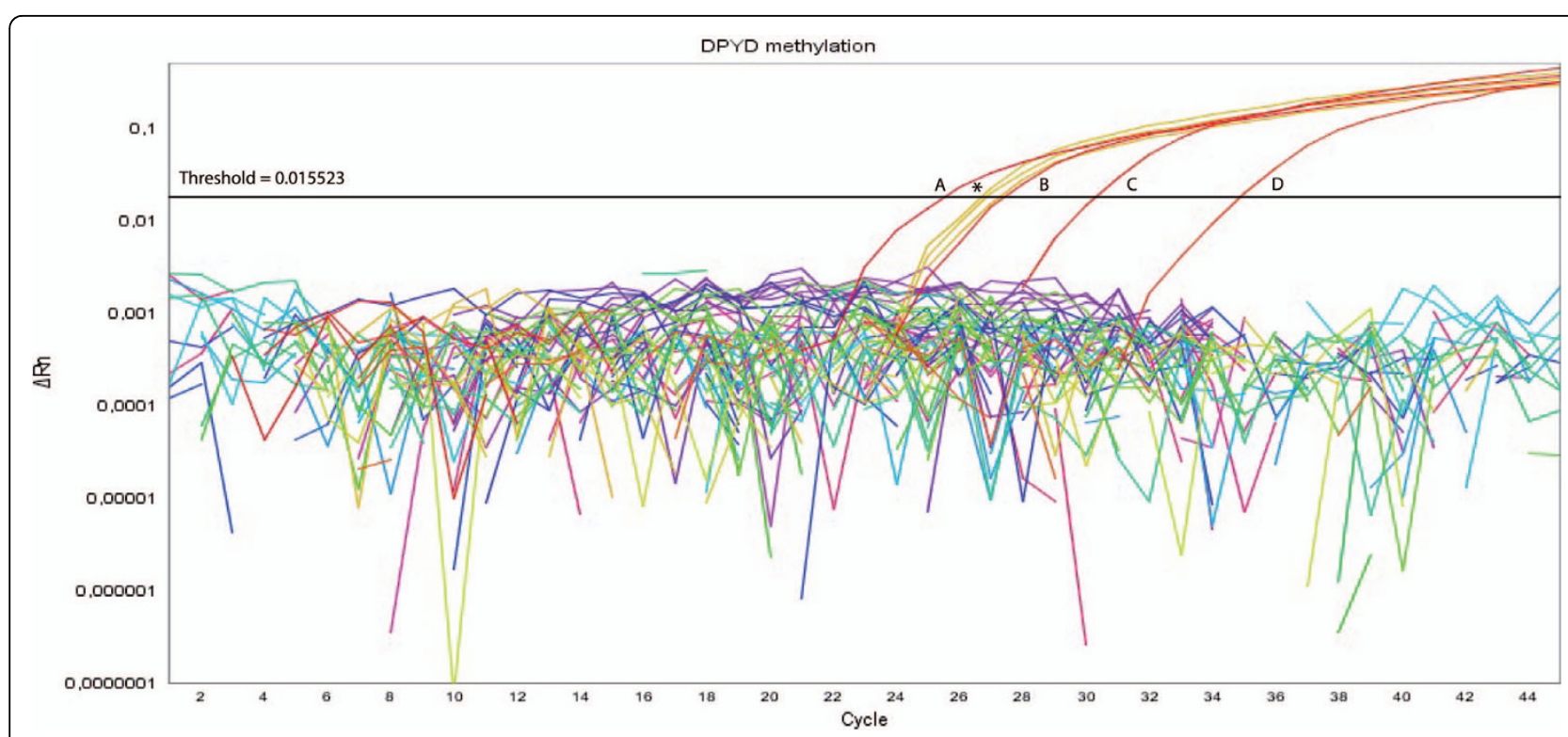

Figure 2 Illustrative QMSP amplification plots for 4 serial 10x-dilutions of fully methylated, bisulfite converted DNA (A, B, C, D) and DPYD promoter methylation in RKO cell line $\left(^{*}\right)$. The DPYD/ACTB ratios were determined using the cycle number where fluorescence per reaction crossed the threshold, which is set to the geometrical phase of polymerase chain reaction amplification above background. $\Delta R n$ is defined as the cycle-to-cycle change in the fluorescence signal (log scale).

in the 29 microdissected tumor tissue samples from patients experiencing severe 5-FU toxicity. Nevertheless, $D P Y D$ promoter methylation was detected in the RKO cell line (methylation ratio $=1075)($ Figure 2$)$.

Concerning mutation analysis, the MLPA assay was performed in 42 of 45 cases (3 cases were not studied due to low quality DNA). We were able to identify (Figure 3) the two exon 14 skipping mutation (IVS14+1G >
A) previously detected by direct sequencing analysis, but not the $1845 \mathrm{G}>\mathrm{T}$ missense mutation. The remaining 39 patients with severe toxicity to 5 -FU treatment that did not carry clinically relevant allelic variants in exon 14 of $D P Y D$, were further screened for large $D P Y D$ intragenic rearrangements. No intragenic rearrangements were found for DPYD in the peripheral blood samples of those patients.

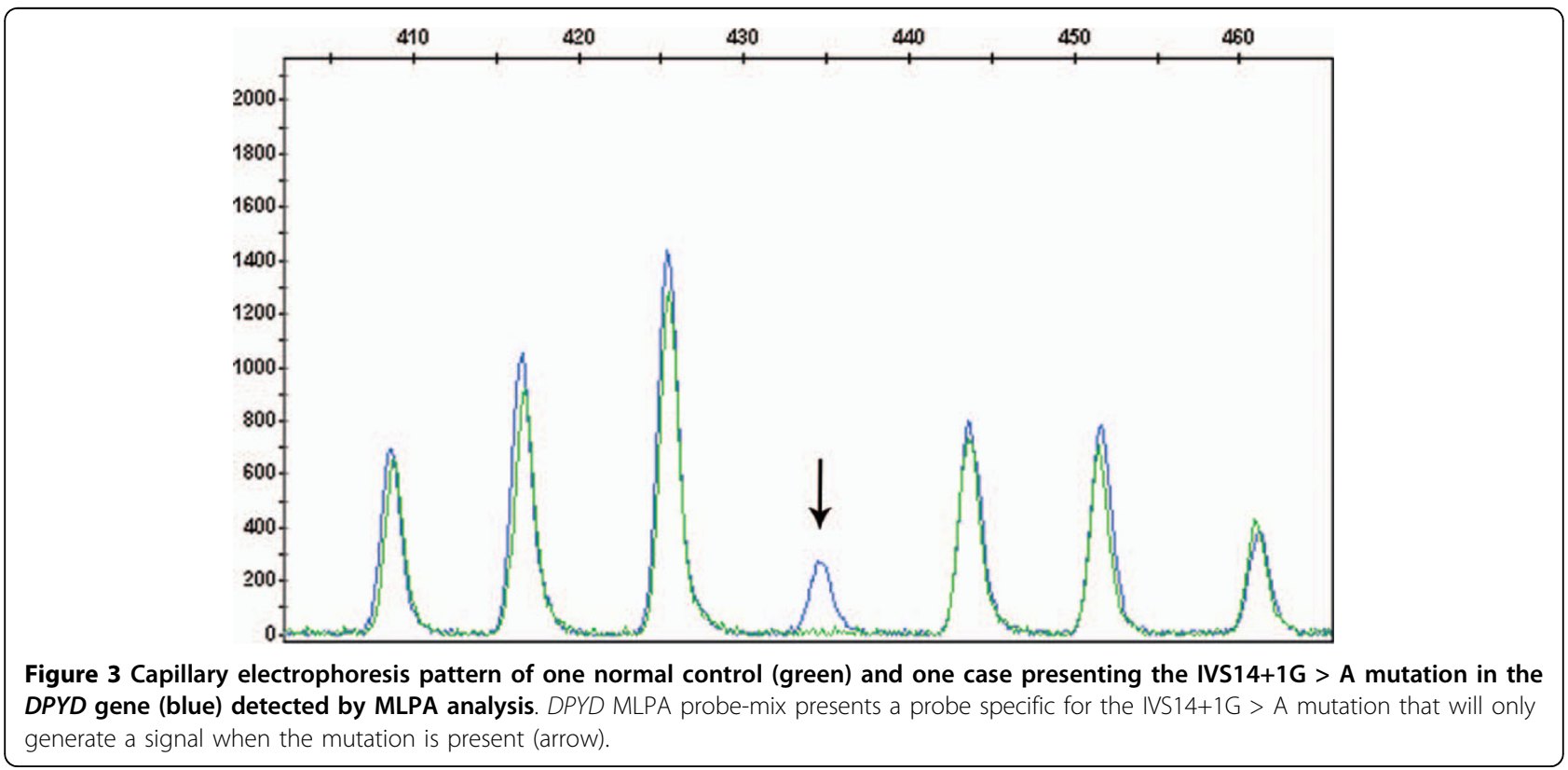




\section{Discussion and Conclusions}

5-FU is broadly used in the treatment of GI cancer. Deficiency of the enzyme DPD, encoded by the DPYD gene, has been associated with the development of severe toxicity to 5-FU in GI cancer patients. Since aberrant promoter methylation has been proposed as an alternative mechanism of DPD deficiency, we assessed the methylation status of $D P Y D$ promoter region by quantitative methylation-specific PCR in GI cancer patients who developed 5-FU-related severe toxicity. Moreover, the analysis of large intragenic rearrangements of DPYD, which have also been causally related with DPD deficiency, was additionally performed.

Severe toxicity associated with 5 -FU has been attributed in a small number of cases to allelic variants of the $D P Y D$ gene, which result in lower DPD enzyme activity. However, in most cases of severe 5-FU toxicity no genetic mechanism has been described. Thus, a possible role for epigenetic alteration of $D P Y D$, especially promoter methylation, has been hypothesized. The first published study on this issue found that methylation of $D P Y D$ promoter in peripheral blood leucocyte DNA from colorectal patients was associated with severe 5-FU toxicity[17]. In our series of 45 patients with GI cancer with severe 5-FU toxicity, methylation at the DPYD promoter was not found in any of the cases, neither in peripheral blood leucocytes nor in tumor tissue samples. Importantly, a well characterized colon cancer cell line (RKO), known to harbor extensive CpG methylation at the $D P Y D$ promoter, tested positive, thus validating the methodology used in our study. Indeed, while this study was being performed, two independent studies reported similar findings in peripheral blood leucocytes of 28 patients[6] and 17 patients[28] with severe toxicity following 5-FU administration. It is noteworthy that the first cited study examined only 15 individuals, of which only five were cancer patients[17], whereas the two latter studies[6,28] and our own comprise a total of 90 patients, Indeed, our study has the largest single series of patients among all cited studies. Thus, our data further sustains that $D P Y D$ promoter methylation is absent in patients treated with 5-FU for GI cancer which have developed severe toxicity.

All patients enrolled in this study were screened for $D P Y D$ exon 14 mutations by sequencing (including the exon 14 skipping mutation IVS14+1G > A), eight of which were included in a previous publication on unselected colorectal cancer patients[29]. This mutation was found in two patients (one previously reported) and both of them developed severe toxicity following 5-FU administration. Previous observations confirmed the high specificity, positive and negative predictive values of this genetic analysis $[6,14,30]$. However, our previous analysis of 73 consecutive colorectal cancer patients detected exon 14 mutations in only two of eight cases with severe toxicity[29].

Both patients harboring the skipping mutation in exon 14 (IVS14+1G > A) and the patient harboring the 1845 $\mathrm{G}>\mathrm{T}$ missense mutation, suffered from grade 4 toxicity (mucositis and febril neutropenia) related to 5-FU chemotherapy [29]. Thus, although DPYD mutational screening identifies cases which are prone to develop 5FU-related toxicity, the low prevalence of those mutations might raise questions regarding the cost-effectiveness of the procedure.

Considering the results obtained for $D P Y D$ promoter methylation and skipping mutation in exon 14 (IVS14+1G $>$ A) analysis, we decided to determine whether large $D P Y D$ intragenic rearrangements might explain 5-FU toxicity. However, those large intragenic rearrangements were also not found in any of the 39 cases, thus excluding a significant role for this genetic alteration in the impairment of DPD activity. This result is also in agreement with a recently published study in which no large rearrangements were found in series of 68 patients experiencing severe 5-FU toxicity[31,32].

In conclusion, considering our own and previously published data, epigenetic silencing and intragenic rearrangements of DPYD do not contribute to the development of severe 5-FU toxicity in GI cancer patients. Although severe 5-FU toxicity is a significant clinical concern, additional studies integrating a more comprehensive analysis of 5-FU metabolic pathway are required to uncover the factors underlying the majority of patients which experience severe 5-FU toxicity.

\section{Acknowledgements}

The authors thank Natália Salgueiro for kindly providing mutational analysis data of eight cases. The contribution of pathologists Carlos Lopes, MD, PhD, Eduardo Ferreira, MD, Isabel Gomez, MD, Lígia Castro, MD, Macedo Dias, MD, Olinda Lima, MD, Silva Caspurro, MD, and Vicente Gonçalves, MD, PhD, which kindly provided representative tumor samples from some cases included in this study, is greatly acknowledged.

This work was supported by a research grant from the Research Center of Portuguese Oncology Institute - Porto (Cl-IPOP 4-2008).

\section{Author details}

'Department of Medical Oncology, Portuguese Oncology Institute - Porto, Portugal. ${ }^{2}$ Department of Genetics, Portuguese Oncology Institute - Porto, Portugal. ${ }^{3}$ Department of Pathology, Portuguese Oncology Institute - Porto, Portugal. ${ }^{4}$ Cancer Epigenetics Group, Research Center of the Portuguese Oncology Institute - Porto, Portugal. ${ }^{5}$ Cancer Genetics Group, Research Center of the Portuguese Oncology Institute - Porto, Portugal. ${ }^{6}$ Department of Pathology and Molecular Immunology, Institute of Biomedical Sciences Abel Salazar, University of Porto, Porto, Portugal.

\section{Authors' contributions}

JS-B collected the clinical data, carried out experiments and drafted the manuscript. JR-C, MP and VLC carried out experiments. AR assisted in the review of the histopathological material. PCD carried out experiments including tissue block sectioning. MM helped with the collection of clinical data. MRT was involved in drafting the manuscript. $\mathrm{RH}$ helped in the design 
of the study, performed the review of the histopathological material and assisted in the finalization of the manuscript. CJ designed and supervised the project, and finalized the manuscript. All authors have read and approved the final version of the manuscript.

\section{Competing interests}

The authors declare that they have no competing interests.

Received: 11 May 2010 Accepted: 1 September 2010

Published: 1 September 2010

\section{References}

1. Longley D, Harkin DP, Johnston P: 5-fluorouracil: mechanisms of action and clinical strategies. Nat Rev Cancer 2003, 3:330-338.

2. Wohlhueter RM, Mclvor RS, Plagemann PG: Facilitated transport of uracil and 5-fluorouracil, and permeation of orotic acid into cultured mammalian cells. J Cell Physiol 1980, 104:309-319.

3. Noordhuis P, Holwerda U, Van der Wilt CL, Van Groeningen CJ, Smid K, Meijer S, Pinedo HM, Peters GJ: 5-Fluorouracil incorporation into RNA and DNA in relation to thymidylate synthase inhibition of human colorectal cancers. Ann Oncol 2004, 15:1025-1032.

4. Perry MC: The Chemotherapy Source Book. Lippincott Williams \& Wilkins, 42008.

5. UpToDate Online 17.1. [http://www.uptodate.com].

6. Schwab M, Zanger UM, Marx C, Schaeffeler E, Klein K, Dippon J, Kerb R, Blievernicht J, Fischer J, Hofmann U, et al: Role of genetic and nongenetic factors for fluorouracil treatment-related severe toxicity: a prospective clinical trial by the German 5-FU Toxicity Study Group. J Clin Oncol 2008, 26:2131-2138

7. Harstrick A, Gonzales A, Schleucher N, Vanhoefer U, Lu K, Formento JL, Milano G, Wilke H, Seeber S, Rustum Y: Comparison between short or long exposure to 5-fluorouracil in human gastric and colon cancer cell lines: biochemical mechanism of resistance. Anti-Cancer Drugs 1998, 9:625-634.

8. Ichikawa W, Uetake H, Shirota Y, Yamada H, Nishi N, Nihei Z, Sugihara K, Hirayama R: Combination of dihydropyrimidine dehydrogenase and thymidylate synthase gene expressions in primary tumors as predictive parameters for the efficacy of fluoropyrimidine-based chemotherapy for metastatic colorectal cancer. Clin Cancer Res 2003, 9:786-791.

9. Soong R, Shah N, Salto-Tellez M, Tai BC, Soo RA, Han HC, Ng SS, Tan WL, Zeps $N$, Joseph D, et al: Prognostic significance of thymidylate synthase, dihydropyrimidine dehydrogenase and thymidine phosphorylase protein expression in colorectal cancer patients treated with or without 5fluorouracil-based chemotherapy. Annals of Oncology 2008, 19:915-919.

10. Ezzeldin HH, Diasio R: Predicting fluorouracil toxicity: can we finally do it? J Clin Oncol 2008, 26:2080-2082.

11. van Kuilenburg $A B$ : Dihydropyrimidine dehydrogenase and the efficacy and toxicity of 5-fluorouracil. Eur J Cancer 2004, 40:939-950.

12. Maekawa K, Saeki M, Saito Y, Ozawa S, Kurose K, Kaniwa N, Kawamoto M, Kamatani N, Kato K, Hamaguchi T, et al: Genetic variations and haplotype structures of the DPYD gene encoding dihydropyrimidine dehydrogenase in Japanese and their ethnic differences. $J$ Hum Genet 2007, 52(10):804-819.

13. Collie-Duguid ES, Etienne MC, Milano G, McLeod HL: Known variant DPYD alleles do not explain DPD deficiency in cancer patients. Pharmacogenetics 2000, 10(3):217-223.

14. Raida $M$, Schwabe $W$, Häusler $P$, van Kuilenburg $A B$, van Gennip $A H$, Behnke D, Höffken K: Prevalence of a common point mutation in the dihydropyrimidine dehydrogenase (DPD) gene within the $5^{\prime}$-splice donor site of intron 14 in patients with severe 5-fluorouracil (5-FU)related toxicity compared with controls. Clin Cancer Res 2001, 7:2832-2839.

15. van Kuilenburg AB, Meijer J, Mul AN, Hennekam RC, Hoovers JM, de DieSmulders CE, Weber P, Mori A, Bierau J, Fowler B, et al: Analysis of severely affected patients with dihydropyrimidine dehydrogenase deficiency reveals large intragenic rearrangements of DPYD and a de novo interstitial deletion del(1)(p13.3p21.3). Hum Genet 2009, 125:581-590.

16. Noguchi T, Tanimoto K, Shimokuni T, Ukon K, Tsujimoto H, Fukushima M, Noguchi T, Kawahara K, Hiyama K, Nishiyama M: Aberrant methylation of DPYD promoter, DPYD expression, and cellular sensitivity to 5fluorouracil in cancer cells. Clin Cancer Res 2004, 10:7100-7107.
17. Ezzeldin HH, Lee AM, Mattison LK, Diasio R: Methylation of the DPYD promoter: an alternative mechanism for dihydropyrimidine dehydrogenase deficiency in cancer patients. Clin Cancer Res 2005, 11:8699-8705.

18. Zhang X, Soong R, Wang K, Li L, Davie J, Guarcello V, Diasio R: Suppression of DPYD expression in RKO cells via DNA methylation in the regulatory region of the DPYD promoter: a potentially important epigenetic mechanism regulating DPYD expression. Biochem Cell Biol 2007, 85:337-346.

19. Common Terminology Criteria for Adverse Events v4.0 (CTCAE). Institute. UDoHaHSNIoHNC 2009.

20. Stanley R, Hamilton LAA: Pathology and Genetics of Tumours of the Digestive System. World Health Organization Classification of Tumours 2000.

21. Pearson $\mathrm{H}$, Stirling D: DNA extraction from tissue. Methods Mol Biol 2003, 226:33-34.

22. Clark SJ, Harrison J, Paul CL, Frommer M: High sensitivity mapping of methylated cytosines. Nucleic Acids Res 1994, 22:2990-2997.

23. Esteller M: Cancer epigenomics: DNA methylomes and histonemodification maps. Nat Rev Genet 2007, 8:286-298.

24. Olek A, Oswald J, Walter J: A modified and improved method for bisulphite based cytosine methylation analysis. Nucleic Acids Res 1996, 24:5064-5066.

25. Dugast-Darzacq C, Grange T: MethylQuant: a real-time PCR-based method to quantify DNA methylation at single specific cytosines. Methods $\mathrm{Mol}$ Biol 2009, 507:281-303.

26. Eads CA, Lord RV, Wickramasinghe K, Long TI, Kurumboor SK, Bernstein L, Peters JH, DeMeester SR, DeMeester TR, Skinner KA, et al: Epigenetic patterns in the progression of esophageal adenocarcinoma. Cancer Res 2001, 61(8):3410-3418.

27. Schouten JP, McElgunn CJ, Waaijer R, Zwijnenburg D, Diepvens F, Pals G: Relative quantification of 40 nucleic acid sequences by multiplex ligation-dependent probe amplification. Nucleic Acids Res 2002, 30(12):e57.

28. Amstutz U, Farese S, Aebi S, Largiadèr C: Hypermethylation of the DPYD promoter region is not a major predictor of severe toxicity in 5fluorouracil based chemotherapy. J Exp Clin Cancer Res 2008, 27:54.

29. Salgueiro N, Veiga I, Fragoso M, Sousa O, Costa N, Pellon M, Sanches E, dos Santos JG, Teixeira M, Castedo S: Mutations in exon 14 of dihydropyrimidine dehydrogenase and 5-Fluorouracil toxicity in Portuguese colorectal cancer patients. Genetics in Medicine 2004, 6:102-107.

30. Omura K: Clinical implications of dihydropyrimidine dehydrogenase (DPD) activity in 5-FU-based chemotherapy: mutations in the DPD gene, and DPD inhibitory fluoropyrimidines. Int J Clin Oncol 2003, 8(3):132-138.

31. Ticha I, Kleiblova P, Fidlerova J, Novotny J, Pohlreich P, Kleibl Z: Lack of large intragenic rearrangements in dihydropyrimidine dehydrogenase (DPYD) gene in fluoropyrimidine-treated patients with high-grade toxicity. Cancer Chemother Pharmacol 2009, 64(3):615-618.

32. Paré L, Paez D, Salazar J, Rio Ed, Tizzano E, Marcuello E, Baiget M: Absence of large intragenic rearrangements in the DPYD gene in a large cohort of colorectal cancer patients treated with a 5FU-based chemotherapy. British Journal of Clinical Pharmacology 2010.

\section{Pre-publication history}

The pre-publication history for this paper can be accessed here: http://www.biomedcentral.com/1471-2407/10/470/prepub

doi:10.1186/1471-2407-10-470

Cite this article as: Savva-Bordalo et al:: Promoter methylation and large intragenic rearrangements of DPYD are not implicated in severe toxicity to 5-fluorouracil-based chemotherapy in gastrointestinal cancer patients. BMC Cancer 2010 10:470. 